\title{
CLOSE BY COMPACT OBJECTS AND RECENT SUPERNOVAE IN THE SOLAR VICINITY
}

\author{
Sergei Popov ${ }^{1,2}$ \\ ${ }^{1}$ University of Padova \\ via Marzolo 8, 35131, Padova, Italy \\ ${ }^{2}$ Sternberg Astronomical Institute \\ Universitetski pr. 13, 119992, Moscow, Russia \\ polar@sai.msu.ru
}

\begin{abstract}
I discuss young close-by compact objects, recent supernovae in the solar neighbourhood, and point to their connection with cosmic ray studies. Especially the role of the Gould Belt is underlined.
\end{abstract}

Keywords: neutron stars, evolution, supernovae, cosmic rays

\section{Introduction}

In this short paper at first I shall try to show links between studies of cosmic rays (CRs) and studies of close-by compact objects (in particular neutron stars - NSs). The reason for an existence of such relation is obvious: both phenomena (CRs and NSs) have the same origin - supernova (SN) explosions. Then I discuss in more details the population synthesis of close-by NSs. The analysis of the population of these sources makes us to conclude that the solar neighbourhood (by that I mean a region about few hundred of parsecs around the Sun) in enriched with young NSs. It is a natural consequence of the existence of the Gould Belt - local structure formed by massive stars.

The main message I want to deliver in this contribution is the following. We are living in a region of the Milky way enriched with massive stars in comparison with a typical place at $8 \mathrm{kpc}$ from the center of the Galaxy. Due to this fact SN rate in $\sim 600 \mathrm{pc}$ around the Sun during the last few tens of Myrs is enhanced. It results also in the ebhanced number of near-by young compact objects. Now we know about two tens of young (age $<4$ Myrs) close (distance $<1 \mathrm{kpc}$ ) NSs. This local (in space and time) increase of SN rate can be important for modeling of CR flux on the Earth. 


\section{Cosmic rays and recent close-by supernovae}

Galactic CRs are genetically connected with SN: CRs are accelerated in SN remnants (see other materials in this volume, especially contributions by Michal Ostrowski, Vladimir Ptuskin and John Wefel). The local CR density is inevitably dependent on the rate of SN in the Galaxy in general and in the solar proximity in particular. CR flux can be increased if the Sun appears in a region of the Galaxy with enhanced SN rate, or if by chance several SN explosions in a row happen in the vicinity of the Sun.

For example Shaviv (2004) suggested, that passages of the Sun through galactic spiral arms are accompanied by increase of CR flux on Earth (at this conference some results were presented in the talk by Smadar Levi). As CRs can have influence on Earth climate (in particular on cloud formation) such passages can be responsible for the (quasi)periodic appearence of ice age epochs on our planet. However, I would like to note, that even local quasiperiodic fluctuations of starformation rate (and correspondent changes in SN rate) can give a similar effect. Such fluctuations were found by Vanbeveren and De Donder (2003) in their modeling of star formation in the solar vicinity. Another possibility to enhance $\mathrm{SN}$ rate is connected with the formation of structures like the Gould Belt (see description of the Belt below). ${ }^{1}$ All these effects can work together, and therefore modeling of the CR flux history becomes a very complicated business because of a "turbulent history" of star formation in $\sim 1 \mathrm{kpc}$ around the Sun.

For a long time local SN were considered as one of candidates for a catastrophic event responsible for mass extinction of species. Still SN potentially can play a "positive" role in the history of life on Earth. An interesting possibility of SN influence on bioworld of our planet can be connected with the hypothesis discussed by Tsarev (1999; it is also described in an easier available paper by Chernavskii 2000). In the original version the author suggested that chirality symmetry breaking existing in the bioorganic world can be connected with strong neutrino burst due to a close SN which happened in the early stages of the history of the Earth. However, a close-by SN (close enough to povide a strong neutrino flux) can just destroy terrestrial bioorganics. In that sense it is possible to suggest a more promising hypothesis which includes so called "choked gamma-ray bursts" (Meszaros, Waxman 2001). If a jet is unable to penetrate through the stellar envelope, then only a neutrino beam will break-out. In that case it is possible both: to increase the neutrino flux, and to decrease negative influence of hard radiation of a SN on the biosphere.

Recent local SN (and related close-by NS) can strongly influence not only the flux of CR but the spectrum as well. Erlykin and Wolfendale (1997) pro-

${ }^{1}$ Effects of the Belt on CR characteristics were discussed in Pohl et al. (2003) and in Dermer (2004). 
posed that a knee in the spectrum is due to a single source. According to their estimates there should exist a near-by relatively young SN remnant. An estimated age of such a hypothetical object was found by these authors to be equal to 80-100 kyr, and its distance - to $230-350 \mathrm{pc}$. They suggested that the Monogem nebula (and related to it PSR 0656+14) can be such a source which produce the knee in the spectrum (see also the contribution by Jorg Hoerandel in this volume for a more detailed discussion of the knee).

How probable an appearence of such an object is? If the Sun is sutuated in a typical region of the Galaxy at $8 \mathrm{kpc}$ from its center then the probability of appearence of a SN remnant of necessary age at necessary distance is very low. However, we are living in a region of enhanced $\mathrm{SN}$ rate, and there are many young compact objects around us. In the following section I am going to discuss this population and it origin in more details.

\section{Young close-by NSs and the Gould Belt}

The solar proximity is rich in young NSs. Some of them represent a new class of sources: radioquiet X-ray dim isolated NSs (see a review in Treves et al. 2000). The reason of such enrichment is an existence of a structure formed by massive stars - the Gould Belt. In the following subsection we discuss it in some details. Then we present new results on a population synthesis of young NSs, and finally we discuss a convenient way to represent this population.

Population of near-by young NSs is presented in the table (reproduced from Popov, Turolla 2004). We included there 20 objects. Thirteen of them are detected as thermal emitters, i.e. as coolers. They are seven radioquiet ROSAT NSs (aka the Magnificent seven - M7), Geminga and geminga-like source 3EG $\mathrm{J} 1835+5918$, and four normal radiopulsars (among which the famous Vela pulsar, and PSR B0656+14 which is probably connected with the single source, see above). Seven close-by NSs which are not detected as thermal emitters (shown at the bottom of the table) are normal radiopulsars with ages 2-4 Myrs.

\section{The Gould Belt}

The Gould Belt is a structure consisting of clusters of massive stars. About $2 / 3$ of massive stars inside $600 \mathrm{pc}$ around the Sun belong to the Belt (see a recent paper by Grenier 2004 for main references). The Gould Belt radius is about $300-500 \mathrm{pc}$. It is inclined at $\sim 18-22^{\circ}$ respect to the galactic plane. The Sun is situated not far from the center of that disc-like structure. The age of the Belt is not well estimated, 30-60 Myrs can be a good guess. The origin of this system is unclear. One of the possibility is an impact of a high velocity cloud with the galactic disk. It is important to note, that the Sun and the Belt are not genetically related to each other. 
Due to the presence of the Belt the rate of SN around us (say in a few hundred parsecs) during last several tens of million years is higher than it is at an average place at a solar distance from the galactic center. The enhancement is about 3-4 times over the Galactic average. Grenier (2003) estimates a SN rate in the Belt as 20-27 per Myr during the last few Myrs. Because of that there should be a local overabundance of young NSs which can appear as hot cooling objects, as gamma-ray sources etc. (in priciple in addition to NSs there should be young BHs in the solar neighbourhood, but these objects are much more elusive; it would be very interesting to detect them, see Prokhorov, Popov 2002). For example, such young NSs can form a subpopulation among EGRET unidentified sources. The existence of the Local Bubble can be also linked with the Belt (Berghofer, Breitwerdt 2002).

\section{$\log N$ - Log S distribution of close-by NSs}

One of the most powerful methods of investigating properties of some population of sources is a $\log \mathrm{N}-\log \mathrm{S}$ distribution. It is an integral distribution, which shows the number of sources with a flux larger than a given value. It is useful to remind two limiting cases. If sources are distributited homogeniously and isotropicaly then the slope of $\log \mathrm{N}-\log \mathrm{S}$ is equal to $-3 / 2$ : the number of sources grows as the cube of a distance, but the observed flux decreases as the square. In the case of a disk-like system the slope is -1 (the number of objects is growing only as a square of a distance). If sources are located at the same distance (for example, sources in a given far-away galaxy) then $\log \mathrm{N}-$ $\log \mathrm{S}$ shows distribution of sources in luminosity.

Popov et al. (2003) calculated Log N - Log S distribution of cooling NSs in the solar vicinity, which can be observed by ROSAT and other X-ray missions. The results are shown in the fig. 1 .

The main output of these calculations can be formulated as follows: observed cooling isolated NSs originated in the Gould Belt. This result does not depend on the uncertainties of the population synthesis model. I want to note, that according to our calculations there should be about several tens more unidetified isolated NSs with fluxes detectable by ROSAT. These objects are hiding in crowded regions close to the galactic plane (and to the plane of the Gould Belt). In addition there should be many NSs with ages above $\sim 1 \mathrm{Myr}$, which now are too cold now to be detected. A total expected number of NSs depending on their ages and distances can be easily shown on the age-distance diagram.

If all uncertainties like initial spatial distribution of NSs, their kick velocity distribution, characteristics of emission (atmospheric effects etc.), mass spectrum of NSs are fixed, then $\log \mathrm{N}-\log \mathrm{S}$ distribution of close-by NSs can be a powerful tool to put constraints on models of thermal evolution of NSs. 
Table 1. Local $(D<1 \mathrm{kpc})$ population of young (age $<4.25$ Myrs) isolated neutron stars

\begin{tabular}{|c|c|c|c|c|c|c|}
\hline Source name & $\begin{array}{c}\text { Period } \\
\mathrm{s}\end{array}$ & $\begin{array}{l}\mathrm{CR}^{a} \\
\mathrm{cts} / \mathrm{s}\end{array}$ & $\begin{array}{c}\dot{P} \\
10^{-15} \mathrm{~s} / \mathrm{s}\end{array}$ & $\begin{array}{c}\mathrm{D} \\
\mathrm{kpc}\end{array}$ & $\begin{array}{l}\text { Age }^{b} \\
\text { Myrs }\end{array}$ & Refs \\
\hline Magnificent seven & & & & & & \\
\hline RX J1856.5-3754 & - & 3.64 & - & $0.117^{e}$ & $\sim 0.5$ & {$[1,2]$} \\
\hline RX J0720.4-3125 & 8.37 & 1.69 & $\sim 30-60$ & - & - & {$[1,3]$} \\
\hline RX J1308.6+2127 & 10.3 & 0.29 & - & - & - & [1.4] \\
\hline RX J1605.3+3249 & - & 0.88 & - & - & - & [1] \\
\hline RX J0806.4-4123 & 11.37 & 0.38 & - & - & - & {$[1,5]$} \\
\hline RX J0420.0-5022 & 3.45 & 0.11 & - & - & - & {$[1,11]$} \\
\hline RX J2143.7+0654 & - & 0.18 & - & - & - & {$[6]$} \\
\hline Geminga type & & & & & & \\
\hline PSR B0633+17 & 0.237 & $0.54^{d}$ & 10.97 & $0.16^{e}$ & 0.34 & [7] \\
\hline 3EG J1835+5918 & - & 0.015 & - & - & - & {$[8]$} \\
\hline Thermal. emit. PSRs & & & & & & \\
\hline PSR B0833-45 & 0.089 & $3.4^{d}$ & 124.88 & $0.294^{e}$ & 0.01 & {$[7,9,10]$} \\
\hline PSR B0656+14 & 0.385 & $1.92^{d}$ & 55.01 & $0.762^{f}$ & 0.11 & {$[7,10]$} \\
\hline PSR B1055-52 & 0.197 & $0.35^{d}$ & 5.83 & $\sim 1^{c}$ & 0.54 & {$[7,10]$} \\
\hline PSR B1929+10 & 0.227 & $0.012^{d}$ & 1.16 & $0.33^{e}$ & 3.1 & {$[7,10]$} \\
\hline Other PSRs & & & & & & \\
\hline PSR J0056+4756 & 0.472 & - & 3.57 & $0.998^{f}$ & 2.1 & {$[10]$} \\
\hline PSR J0454+5543 & 0.341 & - & 2.37 & $0.793^{f}$ & 2.3 & {$[10]$} \\
\hline PSR J1918+1541 & 0.371 & - & 2.54 & $0.684^{f}$ & 2.3 & {$[10]$} \\
\hline PSR J2048-1616 & 1.962 & - & 10.96 & $0.639^{f}$ & 2.8 & {$[10]$} \\
\hline PSR J1848-1952 & 4.308 & - & 23.31 & $0.956^{f}$ & 2.9 & [10] \\
\hline PSR J0837+0610 & 1.274 & - & 6.8 & $0.722^{f}$ & 3.0 & {$[10]$} \\
\hline PSR J1908+0734 & 0.212 & - & 0.82 & $0.584^{f}$ & 4.1 & [10] \\
\hline
\end{tabular}

${ }^{a}$ ) ROSAT PSPC count rate

$\left.{ }^{b}\right)$ Ages for pulsars are estimated as $P /(2 \dot{P})$,

for RX J1856 the estimate of its age comes from kinematical considerations.

$\left.{ }^{c}\right)$ Distance to PSR B1055-52 is uncertain $(\sim 0.9-1.5 \mathrm{kpc})$

${ }^{d}$ ) Total count rate (blackbody + non-thermal)

$\left.{ }^{e}\right)$ Distances determined through parallactic measurements

${ }^{f}$ ) Distances determined with dispersion measure

[1] Treves et al. (2000) ; [2] Kaplan et al. (2002); [3] Zane et al. (2002);

[4] Hambaryan et al. (2001); [5] Haberl, Zavlin (2002); [6] Zampieri et al. (2001);

[7] Becker, Trumper (1997); [8] Mirabal, Halpern (2001); [9] Pavlov et al. 2001;

[10] ATNF Pulsar Catalogue (see Hobbs et al. 2003); [11] Haberl et al. 2004 


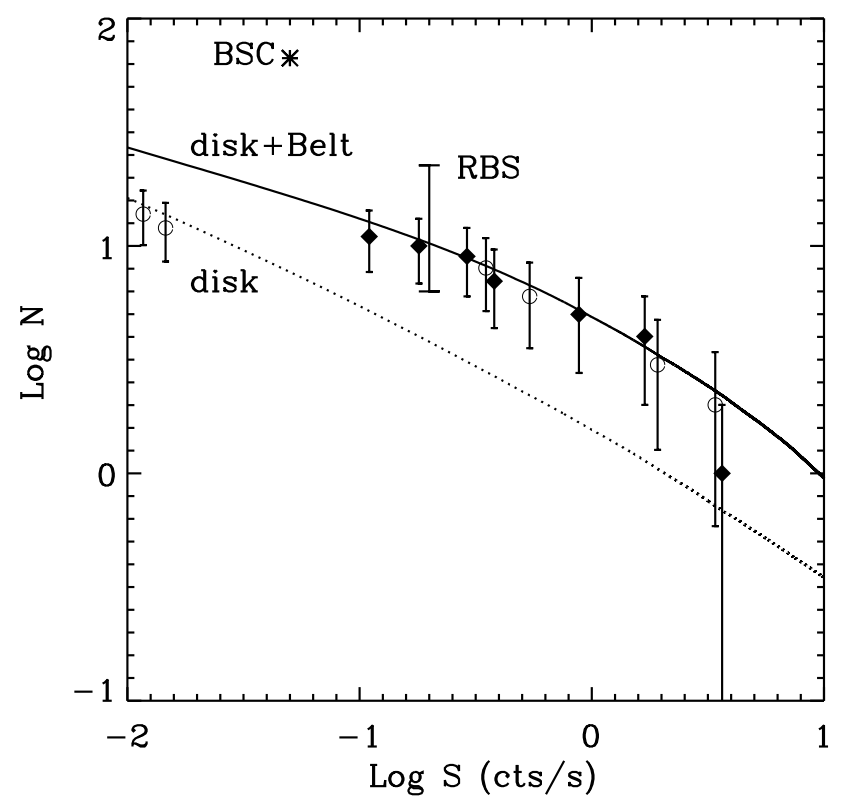

Figure 1. $\log \mathrm{N}-\log \mathrm{S}$ distribution for close-by cooling isolated NSs. Black diamonds are plotted if the dimmest source at specified flux is one of the "Magnificent seven". Otherwise we plot an empty circle. Two lines represent results of calculation. Dotted line - only stars from the galactic disc contribute to the $\log \mathrm{N}-\log \mathrm{S}$ distribution. Solid line - contribution of the Gould Belt is added. "RBS" and "BSC" are two observational limits, obtained from the ROSAT data (RBS: Schwope et al. 1999; BSC: Rutledge et al. 2003). From Popov et al. (2003).

\section{Age-distance diagram}

To illustrate the population of young close-by NSs it is convenient to use the age-distance diagram (Popov 2004).

If we are speaking about observations of thermal emission of young cooling NSs then most of their properties depend on their age, and their detectability in X-rays obviously strongly depends on the distance (not only because of the flux dilution, but also because of the strong interstellar absorption of soft Xrays). In that sense it is useful to plot an age-distance diagram (ADD) for these objects.

There are several reasons to introduce ADDs. First an ADD easily illustrates distributions of sources in age and distance. Then it is possible to plot "expectation lines" for an abundance of sources of different types and compare them with data (i.e. line which show how many sources of smaller than a given value we can expect to find at distances smaller than a given one). Finally since 


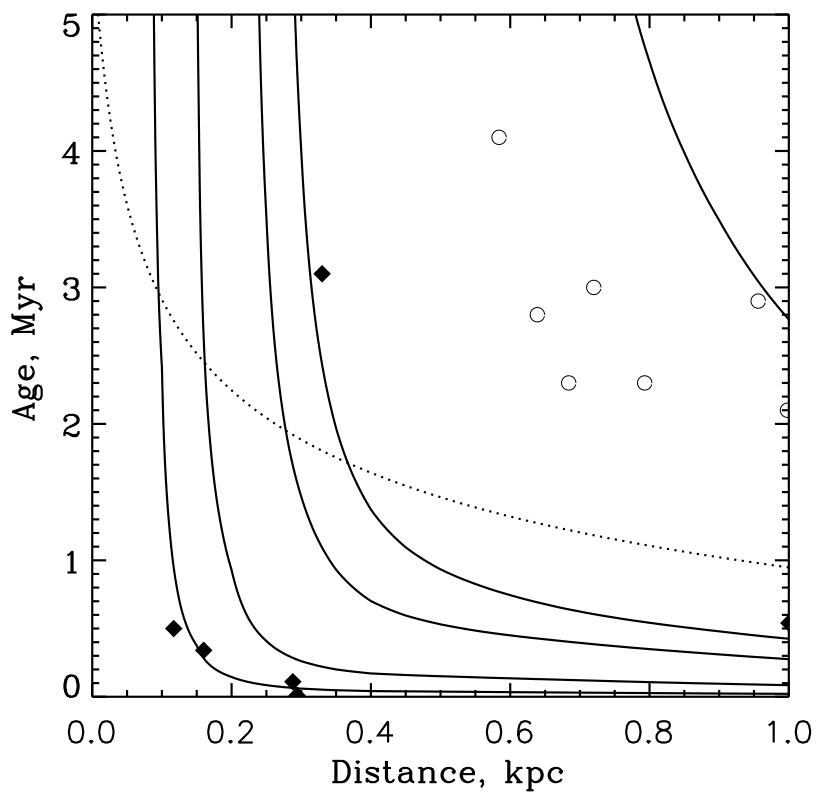

Figure 2. Age-distance diagram with dynamical effects. Solid lines (from bottom to the top) corresponds to 1, 4, 13, 20 and 100 sources. The dotted line is the "visibility" line (see details in Popov 2004).

the observability of sources depends mainly on their ages and distances, it is possible to illustrate observational limits.

In the fig. 2 an ADD for close-by young NSs is presented. These objects can be observed in soft X-rays due to their thermal emission. The thermal evolution strongly depends on the mass. According to different models (see Kaminker et al. 2002, Blaschke et al. 2004 and references there in) a NS with a mass $\sim 1.3-1.4 M_{\odot}$ remains hot $\left(T \sim(0.5-1) 10^{6} \mathrm{~K}\right.$, or equivalently $\sim 50$ $100 \mathrm{eV}$ ) up to $\sim 0.5-1 \mathrm{Myr}$. It corresponds to a luminosity $\sim 10^{32} \mathrm{erg} \mathrm{s}^{-1}$. Usually NSs with low masses $\left(1-1.3 M_{\odot}\right)$ cool down slower, while with higher masses faster.

To plot an ADD it is necessary to fix maximal values for age and distance for selection of sources. I choose 4.25 Myrs as a limiting age for selection of observed sources. This is the time after which even a low-mass NSs cools down to $\sim 10^{5} \mathrm{~K}$ and becomes nearly undetectable in X-rays (see Kaminker et al. 2002, Popov et al. 2004). A limiting distance is taken to be equal to 1 kpc (because of the absorption it is difficult to detect in X-rays an isolated NS at larger distance unless the star is very hot). There are 20 sources of different nature which are supposed to be young close-by NSs (age $<4.25 \mathrm{Myr}$, distance 
$<1 \mathrm{kpc}$ ) (see Popov et al. 2004 and the table above): the M7, Geminga and the geminga-like source RX J1836.2+5925, four PSRs with detected thermal emission (Vela, PSR 0656+14, PSR 1055-52, PSR 1929+10²), and seven PSRs without detection of thermal emission. Not for all of these sources there are good estimates of ages and/or distances (especially for the M7). In the figure there are data points for 13 objects for which such estimates exist (distance to PSR B1055-52 is uncertain, and we accept it to be $1 \mathrm{kpc}$ ).

Note, that there are also two PSRs with distance $<1 \mathrm{kpc}$ and with ages in between 4.25 and 5 Myr (PSR B0823+26, PSR B0943+10) and PSR J0834-60 with distance $0.49 \mathrm{kpc}$ and unknown age. These three objects are not included into the figures. Also PSR B1822-09 with age 0.23 Myr and distance $1 \mathrm{kpc}$ is not plotted.

Two types of objects are distinguished on the graph: detected ones (shown as black diamonds) and undetected due to the thermal X-ray emission (remember, there are at least seven additional sources - six from the M7 and one geminga-like object - for which there are no definite determinations of age or/and distance).

In the picture the "expectation" lines are shown with an inclusion of the dynamical evolution. I.e. here NSs' movements in the Galactic potential are accurately calculated. To plot them we use the same model of progenitor distribution and SN rate as in (Popov et al. 2003). At birth NSs obtain kick velocities in accordance with Arzoumanian et al. (2002) model, and move in the galactic potential.

Five "expectation" lines (solid) are plotted for 1, 4, 13, 20 and 100 sources. For example each point on the line for 20 sources shows us values of age $\left(t_{20}\right)$ and distance $\left(R_{20}\right)$ such that according to our model we expect to see 20 sources with ages less than $t_{20}$ at distances less than $R_{20}$.

The dotted line represents the "visibility" line. The idea of adding such a line is to show the maximal distance for a given age (or vice versa a maximal age for a given distance) at which a hot (i.e. low-mass) NS can be detected. To calculate this line I take the cooling curve from (Kaminker et al. 2002) for $M=1-1.3 M_{\odot}$ (in the model of these authors cooling of NSs with $M<1.35 M_{\odot}$ is nearly mass-independent). Such curves were used for example in (Popov et al. 2003). As soon as a cooling curve is fixed then the age determines the luminosity of the object. The limiting unabsorbed flux is assumed to be $10^{-12} \mathrm{erg} \mathrm{cm}^{-2} \mathrm{~s}^{-1}$. According to WebPIMMS ${ }^{3}$ it corresponds to $\sim 0.01$ ROSAT PSPS counts per second for $N_{H}=10^{21} \mathrm{~cm}^{-2}$ and a blackbody spectrum with $T=90 \mathrm{eV}$, or to $\sim 0.1$ ROSAT PSPS counts per second

\footnotetext{
${ }^{2}$ It should be mentioned, that soft X-ray emission of PSR $1929+10$ can be due to polar caps or due to non-thermal mechanism.

${ }^{3}$ http://heasarc.gsfc.nasa.gov/docs/corp/tools.html
} 
for $N_{H}=10^{20} \mathrm{~cm}^{-2}$ and a blackbody spectrum with $T=50 \mathrm{eV}$. The latter values corresponds to the dimmest source among the M7 - RX J0420.0-5022; the former to possibly detectable hot far away objects. Without any doubt such a simplified approach underestimates the absorption at large distances. So the age at which a NS is still observable is overestimated, but for distances $<1 \mathrm{kpc}$ and ages $<1$ Myr it should not be a dramatic effect.

The fact that the line for 20 sources lies below $\sim 1 / 2$ of the observed points tells us that according to our model not all NSs are observed. For example, at $\mathrm{R}=1 \mathrm{kpc}$ we expect to see 20 sources with ages $\sim 0.5 \mathrm{Myr}$, but in reality we observe 20 sources with age $<4.25 \mathrm{Myr}$. It means that for a constant NS formation rate we see only about $10 \%$ of them. One has to bear in mind that for seven sources ages or/and distances are unknown, but they are suspected to be young and close, this is why here we discuss 20 and not 13 sources. Their inclusion or exclusion changes our conclusions quantitatively, but not qualitatively. Six of the M7 sources which are not plotted on the graphs due to lack of good distance measurement should populate the left bottom corner of the graphs, since according to models of NS thermal evolution (see for example Kaminker et al. 2002, Blaschke et al. 2004) their ages are expected to be $<1 \mathrm{Myr}$, and their distances should be $<500 \mathrm{pc}$. NSs can easily escape detection as coolers simply because they cooled down in $\sim 1$ Myr. A fraction of PSRs can be undetected due to beaming (however, they may be observed as EGRET sources).

PSR B1929+10 lies above the "visibility line". However it is unclear if Xray emission of this faint $\left(0.012\right.$ ROSAT $\left.\mathrm{cts} \mathrm{s}^{-1}\right)$ source is due to non-thermal mechanism or not (Becker \& Trumper 1997). Now this object is not widely accepted as a cooler and usually it is not plotted on the $T-t$ (temperatureage) graphs with cooling curves (see for example Kaminker et al. 2002 and Blaschke et al. 2004).

Surprisingly fluctuations in the part of the plot with small number of NSs (bottom left) are not large: four detected coolers lie just below the line for four sources. Probably we see nearly all low-massive NSs with ages $<1 \mathrm{Myr}$ at $R<300-400 \mathrm{pc}$. It should be noted that we are very lucky to have such a young and close object as the Vela pulsar. If one believes in the mass spectrum with small fraction of NSs with $M>1.5 M_{\odot}$ then inevitably one has to conclude that Vela cannot be a massive NS. It can be important in selection of cooling models (see Blaschke et al. 2004). If in a model Vela is explained only by a cooling curve for $M>1.5 M_{\odot}$ then the model may be questionable. In fact, having such a young and massive NS so close is very improbable. A similar conclusion can be made for Geminga and PSR 0656+14.

There is a deficit of sources below the "visibility" line. These sources could be already detected as dim X-ray sources by ROSAT but were not identified as isolated NSs. The limit for the number of isolated NSs from the BSC (Bright 
Source Catalogue) is about 100 sources at ROSAT count rate $>0.05$ cts s$^{-1}$ (see Rutledge et al. 2003). However we do not expect to see so many young isolated NSs due to their thermal emission. An expected number of coolers observable by ROSAT is about 40 objects with ages $<1$ Myr inside $R<0.5-$ $1 \mathrm{kpc}$ (see the "visibility" line in the fig.2 in comparison with the line for 20 sources, for example). As it was shown by Popov et al. (2003) most of unidentified coolers in ROSAT data are expected to be located at low galactic latitudes in crowded regions.

According to the classical picture all NSs were assumed to be born as PSRs more or less similar to Crab. Depending on the initial parameters they were assumed to be active for $\sim 10^{7}$ yrs. In the last years this picture was significantly changed. Without any doubts we see a deficit of young PSRs in the solar vicinity in comparison with an expected value of young NSs from the Gould Belt and the beaming factor cannot be the only reason for this deficit. This deficit can be explained if one assumes that a significant part of NSs do not pass through the radio pulsar stage or that this stage is extremely short for them (long periods of NSs from the Magnificent seven prove it). Of course fluctuations (in time and space) of NS production rate can be important as far as statistics is not that high.

I conclude this subsection with a note that an ADD can be the useful tool for an illustration of the properties of close-by NSs. Its modifications can be applied to other types of sources. For example an addition of the third axis (for $p$ or $\dot{p}$ for example) can be useful in discussing the population of radiopulsars.

\section{Conclusions}

I want to stress once again that the region of $\sim$ few hundred parsecs around the Sun is characterized by an enhanced SN rate. This is due to the Gould Belt. The Belt (as a structure formed by bright stars) was well known since 19th century. In addition now we observe young close-by NSs which, as it was shown by population synthesis calculations, originated in the Belt. Some of EGRET unidentified sources also are suspected to be young NSs born in the Belt.

The existence of the Gould Belt should inevitably influence properties of CRs detected on Earth.

\section{Acknowledgments}

I thank the Organizers for hospitality and partial financial support. Special thanks to many participants for stimulating discussions. I thank Mirian Tsulaia for comments on the text. 


\section{References}

Arzoumanian, Z., Chernoff, D.F., Cordes, J.M. 2002). "The velocity distribution of isolated radio pulsars", ApJ 568, 289.

Becker, W., Trumper, J. (1997). "The X-ray luminosity of rotation-powered neutron stars", A\&A 326, 682.

Berghofer, T.W., Breitschwerdt, D. (2002). "The origin of the young stellar population in the solar neighborhood - A link to the formation of the Local Bubble?", A\&A 390, 299.

Blaschke, D., Grigorian, H., Voskresensky, D. (2004). "Cooling of neutron stars. Hadronic model.", A\&A (in press) [astro-ph/0403170].

Chernavskii, D.S. (2000), "The origin of life and thinking from the modern physics point of view”, Physics Uspekhi 170, 157.

Dermer, C.D. (2004). "Gamma ray bursts, sepernovae, and cosmic ray origin", in: Proc. of ISCRA 13th Course, Eds. M. Shapiro, T. Stanev and J. Wefel, World Scientific Publ., p.189.

Erlykin, A.D., Wolfendeil, A.W. (1997). "A single source of cosmic rays", J. Phys. G 23, 979.

Grenier, I.A. (2003). "Unidentified EGRET sources in the Galaxy”, astro-ph/0303498.

Grenier, I.A. (2004). "The Gould Belt, star formation, and the local interstellar medium”, astro$\mathrm{ph} / 0409096$.

Haberl F., Zavlin V. (2002). "XMM-Newton observations of the isolated neutron star RX J0806.44123 ", A\&A 391, 571.

Haberl, F. et al. (2004). "The isolated neutron star X-ray pulsars RX J0420.0-5022 and RX J0806.4-4123: New X-ray and optical observations”, A\&A 424, 635.

Hambaryan, V., Hasinger, G., Schwope, A. D., Schulz, N. S. (2001). "Discovery of 5.16 s pulsations from the isolated neutron star RBS 1223", A\&A 381, 98.

Hobbs, G. Manchester, R. Teoh, A. Hobbs, M. (2003). "The ATNF Pulsar Catalogue”, in: Proc. of IAU Symp. 218. "Young neutron stars and their environment" [astro-ph/0309219].

Kaminker, A.D., Yakovlev, D.G., Gnedin, O.Y. (2002). "Three types of cooling superfluid neutron stars: theory and observations", A\&A 383, 1076.

Kaplan, D.L., van Kerkwijk, M.H., Anderson, J. (2002). "The parallax and proper motion of RX J1856.5-3754 revisited", ApJ, 571, 447.

Meszaros, P., Waxman, E. (2001). "TeV Neutrinos from Successful and Choked Gamma-Ray Bursts", Phys. Rev. Lett. 87, 171102.

Mirabal, N., Halpern, J.P. (2001). "A neutron star identification for the high-energy gamma-ray source 3EG J1835+5918 detected in the ROSAT All-Sky Survey", ApJ 547, L137.

Pavlov, G. et al. (2001). "The X-ray spectrum of the Vela pulsar resolved with the Chandra X-ray observatory", ApJ 552, L129.

Pohl, M., Perrot, C., Grenier, I., Digel, S. (2003). "The imprint of Gould's Belt on the local cosmic-ray electron spectrum", A\&A 409, 581.

Popov, S.B., Turolla, R., Prokhorov, M.E., Colpi, M., Treves, A. (2003). "Young close-by neutron stars: the Gould Belt vs. the galactic disc", astro-ph/0305599.

Popov, S.B., Turolla, R., Prokhorov, M.E., Colpi, M., Treves, A. (2004). "Young compact objects in the solar vicinity", in: Proc. of ISCRA 13th Course, Eds. M. Shapiro, T. Stanev and J. Wefel, World Scientific Publ., p.101.

Popov, S.B., Turolla, R. (2004). "Isolated neutron stars: An astrophysical perspective", in: Proc. of the NATO Advanced Research Workshop On Superdense QCD Matter and Compact Stars (in press) [astro-ph/0312369].

Popov, S.B. (2004). “Age-Distance diagram for close-by young neutron stars”, astro-ph/0407370.

Prokhorov, M.E., Popov, S.B. (2002). "Close young isolated black holes”, Astronomy Letters 28. 609. 
Rutledge, R.E., Fox, D.W., Bogosavljevic, M., Mahabal, A. (2003). "A limit on the number of isolated neutron stars detected in the ROSAT Bright Source Catalogue", astro-ph/0302107.

Schwope, A.D., Hasinger, G., Schwarz, R., Haberl, F., Schmidt, M. (1999). "The isolated neutron star candidate RBS 1223 (1RXS J130848.6+212708)", A\&A 341, L51.

Shaviv, N. (2004). "Cosmic ray diffusion in the Milky Way: model, measurement and terrestrial effects", in: Proc. of ISCRA 13th Course, Eds. M. Shapiro, T. Stanev and J. Wefel, World Scientific Publ., p.113.

Treves, A., Turolla, R., Zane, S., Colpi, M. (2000). "Isolated neutron stars: accretors and coolers", PASP 112, 297.

Tsarev, V.A. (1999). "Chiral effects of neutrino after supernova explosions", Bulletin of the Lebedev Physics Institute 2, 22.

Vanbeveren, D., De Donder, E. (2003). "The effect of the process of star formation on the temporal evolution of the WR and O-type star populations in the Solar neighbourhood", astro-ph/0309104.

Zampieri, L. et al. (2001). “1RXS J214303.7+065419/RBS 1774: a new isolated neutron star candidate", A\&A 378, L5.

Zane, S. et al. (2002). "Timing analysis of the isolated neutron star RX J0720.4-3125", MNRAS $334,345$. 